\title{
Transient Photovoltage in Perovskite Solar Cells: Interaction of Trap- Mediated Recombination and Migration of Multiple Ionic Species
}

\author{
Daniel Walter*1, Andreas Fell ${ }^{2,3}$, Yiliang $\mathrm{Wu}^{1}$, The Duong ${ }^{1}$, Chog Barugkin ${ }^{1}$, Nandi $\mathrm{Wu}^{1}$, Thomas \\ White $^{1}$, Klaus Weber ${ }^{1}$ \\ ${ }^{1}$ Research School of Engineering, Australian National University, Canberra, Australia \\ ${ }^{2}$ Fraunhofer Institute for Solar Energy Systems, Freiburg, Germany \\ ${ }^{3}$ AF simulations, Landstr. 12, 79232 March, Germany \\ *Corresponding author: daniel.walter@anu.edu.au
}

Abstract - It is highly probable that perovskite solar cells (PSCs) are mixed electronic-ionic conductors, with ion migration the driving force for PSC hysteresis. However, there is much that is not understood about the interaction of ion migration with other processes in the cell. The key question is: what factors of a PSC are influenced when ions are free to move? In this contribution, we employ a numerical drift-diffusion model of PSCs to show that the migration of both anions and cations in interaction with trap-mediated recombination in the bulk and/or at the surfaces of the perovskite absorber can manifest both current-voltage hysteresis and unusual non-monotonic PSC photovoltage transients. We identify that a key mechanism of this interaction is the influence of the net ionic charge throughout the perovskite bulk-which varies as the ions approach new steadystate conditions - on the distribution of electrons and holes and subsequently spatial distribution of trap-mediated recombination modeled after Shockley Read Hall (SRH) statistics. Relative to intrinsic recombination mechanisms, SRH recombination can be highly sensitive to local asymmetries of the electron-hole population. We show that this sensitivity is key to replicating nonmonotonic transients with multiple time constants, the forms of which may have suggested multiple processes. This work therefore supports the conceptualization of the hysteretic behavior of PSCs as dominated by the interplay between ion migration and trap-mediated recombination throughout the perovskite absorber. 


\section{INTRODUCTION}

One of the remarkable features of perovskite solar cells (PSCs) is an often slow (seconds to minutes) relaxation to steady-state following an extrinsic excitation, which most notably manifests as current density-voltage $(\mathrm{J}-\mathrm{V})$ hysteresis ${ }^{1,2}$. There is now significant theoretical and experimental ${ }^{3-8}$ evidence that $^{-}$ supports the hypothesis that mobile ionic charge is a fundamental contributor to hysteretic behavior. For example, $\mathrm{I}^{-}$anions and $\mathrm{MA}^{+}$cations are likely mobile in $\mathrm{MAPbI}_{3}$ perovskites ${ }^{6}$. However, it has yet to be conclusively established what factors of a PSC are influenced when ions are free to move, and how they interact to produce hysteretic phenomena at the device level.

It is highly probable that the influence of ions on the conduction of electrons and holes in the perovskite absorber is a major source of hysteresis. This is supported by several cell-level simulation studies, which have demonstrated that current density-voltage $(\mathrm{J}-\mathrm{V})$ hysteresis requires - in addition to mobile ionselectron-hole recombination somewhere in the perovskite layer ${ }^{9-14}$. However, there is disagreement on where in the perovskite recombination must occur. It has been claimed that recombination isolated to the perovskite-transport layer interface is the most likely cause for $\mathrm{J}-\mathrm{V}$ hysteresis ${ }^{9-11}$, while other approaches have demonstrated J-V hysteresis with uniform distributions of bulk traps ${ }^{12}$. Recently, it was demonstrated that bulk diffusion length, instead of, or in addition to, interface recombination is a significant factor in JV hysteresis ${ }^{13}$. Together, these results describe a theory of J-V hysteresis in which the arrangement of ions under different bias conditions affects recombination and charge extraction, a dependence that becomes weaker the longer the average diffusion length, as determined by the electron-hole mobility and lifetime. 


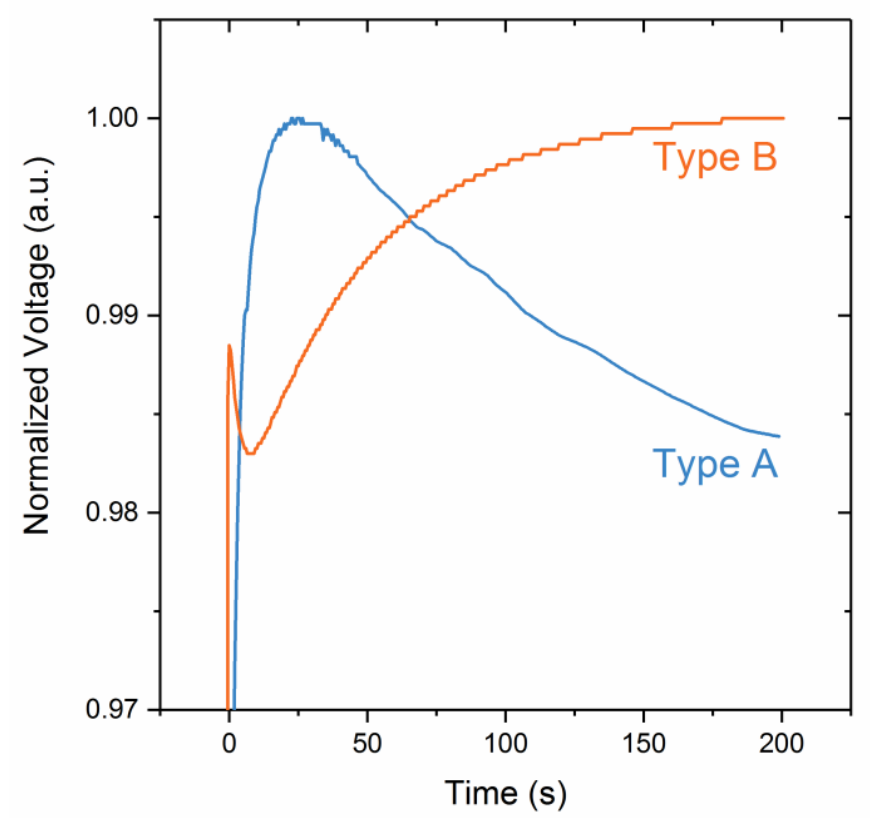

Figure 1: Experimental data exemplary of the two characteristic types of open-circuit voltage transients

However, J-V hysteresis is not the only cell-level manifestation of presumed ion migration in PSCs. For example, we often observe a slow stabilization of voltage or current following a change in illumination or bias conditions in hysteretic PSCs ${ }^{10,11,15-19}$. For this work, we are concerned in particular with notable non-monotonic open-circuit voltage transients (OCVTs) following illumination (figure 1). This response is repeatable, and generally appears in two forms. Type A OCVT has been observed in a range of perovskite mixtures, commonly with cells that are subject to some level of degradation, or are otherwise modestly efficient ${ }^{11,17}$. Type B OCVT, on the other hand, is regularly observed with our laboratory cells based on a quadruple-cation mixture incorporating rubidium, which is being developed for wide band-gap tandem applications ${ }^{20,21}$. Some theories for type A OCVT have been proposed in the literature, including asymmetric recombination in or at the transport layers ${ }^{10}$ and ion-induced changes in energy barriers at the perovskite interfaces affecting charge injection from the transport layers ${ }^{11}$. The type $\mathrm{B}$ response has not yet been extensively investigated. It is not clear, therefore, if the simultaneous presence of non-monotonic voltage transients and $\mathrm{J}-\mathrm{V}$ hysteresis are the result of the same or different processes. Indeed, it is tempting 
to assume that multiple processes must be in play to produce the type B response, given the multiple time constants ${ }^{16,22}$.

In this work we show that a relatively simple physical model can simultaneously replicate $\mathrm{J}-\mathrm{V}$ hysteresis and both types of OCVTs. The key contribution, however, is the observation that replication only requires SRH recombination, in the bulk and/or isolated at the perovskite interfaces, in addition to mobile ions. Our model notably excludes many theories of ion interaction with PSCs, including ions directly influencing the local trap density ${ }^{23}$ and bulk effects in the transport layers ${ }^{18}$. Our work, of course, cannot exclude the validity of these theories, but it demonstrates that they are not be required to replicate the non-monotonic OCVTs.

This work investigates transient migration of both anions and cations. We demonstrate how in the presence of trap-mediated recombination, the ratio of diffusion coefficients of the two ion populations is heavily influential on the transient voltage response, potentially extending the final ion steady state and therefore the voltage transient by many hours or even days. The strong sensitivity of the transient response to the SRH recombination rate, and the concentration and diffusivity of the ion population(s) indicates a possible diagnostic value for these measurements. However, the purpose of the following work is not to achieve precise quantitative agreement with experimental data, but instead to demonstrate how a relatively simple model of a mixed electronic-ionic perovskite semiconductor can replicate these unusual transient responses and describe a common underlying cause.

\section{METHODS}

\section{Open-circuit transient voltage measurements}

The open-circuit voltage transients (OCVTs) were measured on complete PSCs following abrupt illumination from dark conditions using blue-light $(445 \mathrm{~nm})$ LEDs at one-sun equivalent intensity. The cells are temperature controlled at $23{ }^{\circ} \mathrm{C}$, and held in a nitrogen atmosphere. As shown in Figure 1, we 
often observe two characteristically distinct forms of non-monotonic OCVTs. The first, type A, consists of a rise and fall of voltage over a period of tens to hundreds of seconds, while type B OCVT exhibits a more rapid rise and fall, followed by a subsequent rise over a similar timescale. To date, we have measured the type $\mathrm{B}$ response only on quadruple-cation perovskite cells incorporating rubidium (e.g. a FA/MA/Cs/Rb-based perovskite) while the type A response is observed on a wide range of mixtures, including $\mathrm{MAPbI}_{3}$-based cells (including with $\mathrm{Sn}$ ), and multiple-cation mixtures that with and without rubidium doping.

As is well known, the extent of the hysteresis is heavily dependent on the pre-bias conditions and the voltage sweep rate ${ }^{24}$. To measure the $\mathrm{J}-\mathrm{V}$ hysteresis with minimal influence from ion migration during the measurement, we have used protocol in which two curves are measured following roughly a five minute bias at open-circuit and short-circuit. The voltage scan rate is set to $10,000 \mathrm{mV} / \mathrm{s}$. Thus, the sweep direction has minimal impact on the measured curves; they are instead distinguished by initial the ionic conditions. Ion accumulation under short-circuit (SC) is the most unfavorable to charge carrier collection and we therefore measure a reduced fill factor in the SC-stabilized curve. This measurement protocol exacerbates the $\mathrm{J}-\mathrm{V}$ hysteresis, and we observe a substantial difference in fill-factor and efficiency for both cell types.

\section{Modelling perovskite solar cells with Quokka3}

We model charge carrier transport in the perovskite absorber after the well-established semiconductor drift-diffusion models used to model perovskite and inorganic (e.g. Si) semiconductors ${ }^{9,10,12,14,25,26}$, but extend the model by drift-diffusion for two additional charge carriers: anions and cations. It is assumed that ions are neither generated nor "recombine". Instead, a fixed, and equal $^{6,11}$ average concentration of anions and cations migrate through the perovskite bulk via drift and diffusion forces. Our thorough numerical implementation fully computes the ionic-electronic Debye layer 
at the transport layer interfaces, without the need for simplifications such as fixed accumulation layers ${ }^{11}$ or asymptotic approximations ${ }^{12}$. The interfaces are impermeable to ion flow, and do not sink or source ions to the bulk. The solution domain is restricted to the perovskite absorber layer, such that the electron and hole transport layers are treated as metal-semiconductor (MS) boundaries with a defined barrier-height and interface recombination. The general SRH formalism is implemented, as described by Shockley and $\operatorname{Read}^{27}$ being able to simulate the influence of photo-doping and long-lived traps ${ }^{15}$. We note that the simplified SRH formalism often applied in perovskite modelling is only technically valid for conditions where the trap density is at least an order-of-magnitude below the charge carrier density ${ }^{27,28}$, which is not the case for many assumptions of trap densities in perovskite modelling to date. However, we choose the simplified SRH formalism for the simulations of this work, as we find that replication of the OCVTs does not require the effects of the general SRH model.

The model is implemented within the "1D detailed solver" of the solar cell simulation software Quokka3 ${ }^{29}$, both as a steady-state and transient solver. While the semiconductor solver of Quokka3 is well established and validated, we validate the addition of ion migration by comparison to recent pre-prints from Courtier et al ${ }^{30,31}$. Figure 2 plots a simulated PSC voltage sweep from $1.2 \mathrm{~V}$ to short-circuit (SC) and back to $1.2 \mathrm{~V}$ at a rate of $100 \mathrm{mV} / \mathrm{s}$, during which a cation population is in continuous flux. Close agreement between the two models is observed. No fitting of any parameter was performed; the model inputs were replicated exactly from reference ${ }^{31}$. The minor disagreement is most likely the result of differing start conditions for the simulation. In reference ${ }^{30}$, all charge carrier concentrations are initially distributed uniformly throughout the bulk, while our model computes a steady-state solution of the initial operating point before the voltage sweep begins. 


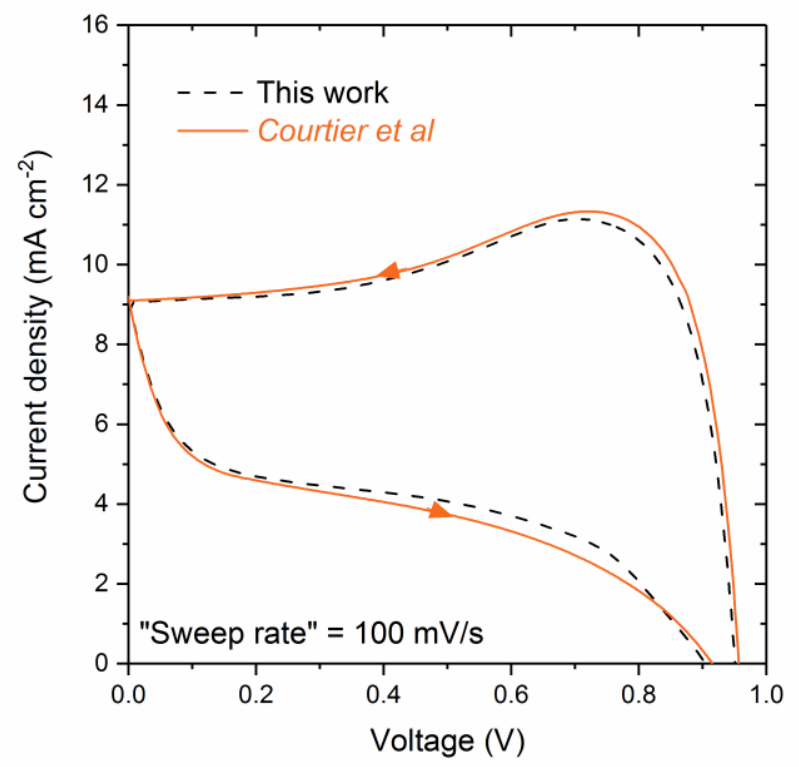

Figure 2: Comparison of this work's simulation of a J-V curve sweep at $100 \mathrm{mV} / \mathrm{s}$ with a similar model $^{30}$. This is a transient simulation, in which a cation population is in continuous flux. Total simulation time was approximately 15 seconds on a $2.3 \mathrm{GHz}$ server-grade Intel CPU (single thread).

To summarize our approach, we note that the model is a simplified implementation of a "perovskite" absorber, which here is represented as a non-excitonic, ambipolar conductor of electrons and holes modelled after the conventional semiconductor drift-diffusion models. The only perovskite-specific feature is thus the inert population of mobile ions. This approach replicates previous works ${ }^{10,11,13}$, and is a reasonable approximation based on our current understanding of the band structure of organic-inorganic perovskites $^{32}$. A summary of the model equations can be found in the supplementary information.

\section{RESULTS AND DISCUSSION}

\section{Open circuit voltage transient measurements}

In Figure 3, we plot the OCVT and J-V curves corresponding to two PSCs that exhibited type A and $\mathrm{B}$ responses. In these examples, the type $\mathrm{A}$ response (Figures $3 \mathrm{a}, 3 \mathrm{~b}$ ) was measured on a standard $\mathrm{MAPbI}_{3}$ perovskite with Spiro-OMeTAD HTL and mp- $\mathrm{TiO}_{2} \mathrm{ETL}$, while the type B response (Figures 3c, 3d) was measured on a quadruple-cation $\mathrm{Rb}$-doped $\mathrm{FA}_{0.75} \mathrm{MA}_{0.15} \mathrm{Cs}_{0.1} \mathrm{PbI}_{2} \mathrm{Br}$ perovskite with a mp-TiO 2 
ETL and PTAA HTL. The fabrication method for the $\mathrm{MAPbI}_{3}$ cell is described in reference ${ }^{33}$, and the quadruple-cation cell in reference ${ }^{20}$.

\section{Simulation of OCVTs}

Transient simulations of PSCs with mobile anions and cations are able to replicate both types of OCVTs. We found that the time-scale and trend of the voltage transient was sensitive to several fundamental material parameters (notably the relative permittivity and transport layer work functions). Because of this, we have avoided overfitting with a multi-parameter fit, and have instead fixed most material parameters (tabulated in supplementary info table S1) and focused qualitatively on the impact of SRH lifetimes, and ion concentration and mobility. We found that the ion parameters (average concentrations and diffusion coefficients) and SRH recombination were the clearest dimensions along which the simulated OCVT could transition between type A and B.

High SRH recombination was necessary to reveal both types of OCVTs. We were able to reproduce the OCVTs with recombination-active traps isolated to the cell interface, uniformly distributed throughout the bulk, or a combination of the two. In Figure 4a, we plot type A OCVTs at a range of bulk trap densities, $N_{t}$, for a device with negligible interface recombination, to show that the magnitude of the voltage deflection is highly sensitive to the rate of SRH recombination. In real cells we expect that there will be a non-zero trap density throughout the device. However, in this work, we choose to focus on conditions where bulk traps (i.e. not interface trapping) are the dominant source of SRH recombination. This is motivated by several factors. Firstly, there has been the relatively limited attention in the perovskite modelling literature to the dynamics of bulk trap recombination, relative to recombination isolated at the interface (see e.g. references ${ }^{9,10,13,18}$ ). Secondly, we found that with dominant interface recombination, the OCVT response was highly sensitive to the work-function of the transport layers (i.e. the barrier height and band bending), which introduced an additional parameter space and complexity that was beyond the 
scope of our study. And finally, we wished to demonstrate how the OCVT response could be replicated in a device without dominant recombination at the transport layer interfaces, to emphasize the fundamental relationship between SRH recombination and ion movement. We stress that the ultimate conclusion of what follows is that the unique dependence of SRH lifetimes to electron-hole asymmetry can be a major influence on the spatial distribution of recombination in PSCs, and key to revealing the experimental OCVTs. This interaction holds for both bulk and/or interface SRH recombination.

However, there is a third form of OCVT that is also observed in the literature. Unlike the type A and $\mathrm{B}$ responses, this form exhibits a slow rise in the open-circuit voltage before stabilizing ${ }^{15,34}$. Our model can replicate this response where non-radiative interface recombination is dominant. In this situation, the movement of ions affects the concentration of minority carriers at the perovskite-transport layer interfaces, and consequently the rate of interface recombination. We discussed this mechanism in a previous study ${ }^{33}$, and a more detailed description of is provided in the supplementary information section S3.

Figure 3 plots the output of the simulations replicating both type A and B voltage transience, and the corresponding $\mathrm{J}-\mathrm{V}$ hysteresis. We first consider the replication of the type A OCVT. Transient simulation studies of PSCs have often considered only a single mobile ion, but DFT calculations of Eames et $a l^{6}$ indicated that both the $\mathrm{I}^{-}$anion and the $\mathrm{MA}^{+}$cation and may be mobile in $\mathrm{MAPbI}_{3}$ perovskites via vacancy assisted diffusion, with diffusion coefficients of $\sim 10^{-12}$ and $\sim 10^{-16}$, respectively (note, this means in our model the more rapid ion is the "cation", being the positively charged $\mathrm{I}^{-}$anion vacancy). Our calculations roughly agree with these values; we found that we could approximate the magnitude and timescale of the experimental OCVT with diffusion coefficients of $5 \times 10^{-11} \mathrm{~cm}^{2} / \mathrm{s}$ and $3 \times 10^{-15} \mathrm{~cm}^{2} / \mathrm{s} \mathrm{for}$ the cation ( $\mathrm{I}^{-}$vacancy) and anion ( $\mathrm{MA}^{+}$vacancy), respectively, although we note that the fitted coefficients depend on many parameters we have not varied, and are indicative only. Thus, it may be more illustrative that we observe good agreement with the data using the same ratio of ion diffusion coefficients as predicted by Eames et al (e.g. $\left.\mathrm{D}_{\text {cation }} / \mathrm{D}_{\text {anion }}=10^{4}\right)$. In fact, we found that the ratio of the diffusivity of the two ion 
populations was profoundly influential on the voltage transience, as illustrated in Figure $4 \mathrm{~b}$. This indicates that the time-scale of OCVTs in PSCs may give some insight into the diffusivity of the ion populations. The initial ion concentration was $10^{19} \mathrm{~cm}^{-3}$, and the bulk trap density was $5 \times 10^{15} \mathrm{~cm}^{-3}$, in the range of reported values for $\mathrm{MAPbI}_{3}{ }^{6,15,35}$. The corresponding $\mathrm{J}-\mathrm{V}$ hysteresis simulations reveal a substantial softening of the fill factor after SC stabilization, a result of reduced charge-carrier collection efficiency. We note that we have not attempted to precisely fit the characteristics of the JV hysteresis, as this also introduces an additional range of fit parameters that are beyond our discussion here. The JV hysteresis simulations are instead presented to illustrate the relative magnitude of the reduced fill factors for conditions replicating type A and B OCVTs. A discussion of the influence of high interface recombination on the JV hysteresis in our model is included in the supplementary information section S2.

To replicate the type $\mathrm{B}$ OCVT, measured on the Rb-doped $\mathrm{FA}_{0.75} \mathrm{MA}_{0.15} \mathrm{Cs}_{0.1} \mathrm{PbI}_{2} \mathrm{Br}^{-b a s e d}$ perovskite, we needed to adjust the respective diffusion coefficients and the ion concentration. In particular, we found that the anion diffusion coefficient needed to be closer to that of the cation; we achieve closest agreement with experiment with cation and anion diffusion coefficients of $8 \times 10^{-14}$ and $3 \times 10^{-}$ ${ }^{15} \mathrm{~cm}^{2} / \mathrm{s}$, respectively, and an ion concentration of $2 \times 10^{17} \mathrm{~cm}^{-3}$. This suggests that, compared to $\mathrm{MAPbI}_{3}$, we require a relatively lower ratio of cation to anion diffusivity, in order to approximate the timescale of the data. Total trap density was $10^{15} \mathrm{~cm}^{-3}$. As with the conditions replicating Type A OCVT, the ion and trap parameters for type B OCVT also replicate the softening fill-factor of the J-V hysteresis, but with a smaller reduction in fill factor between the two curves. 

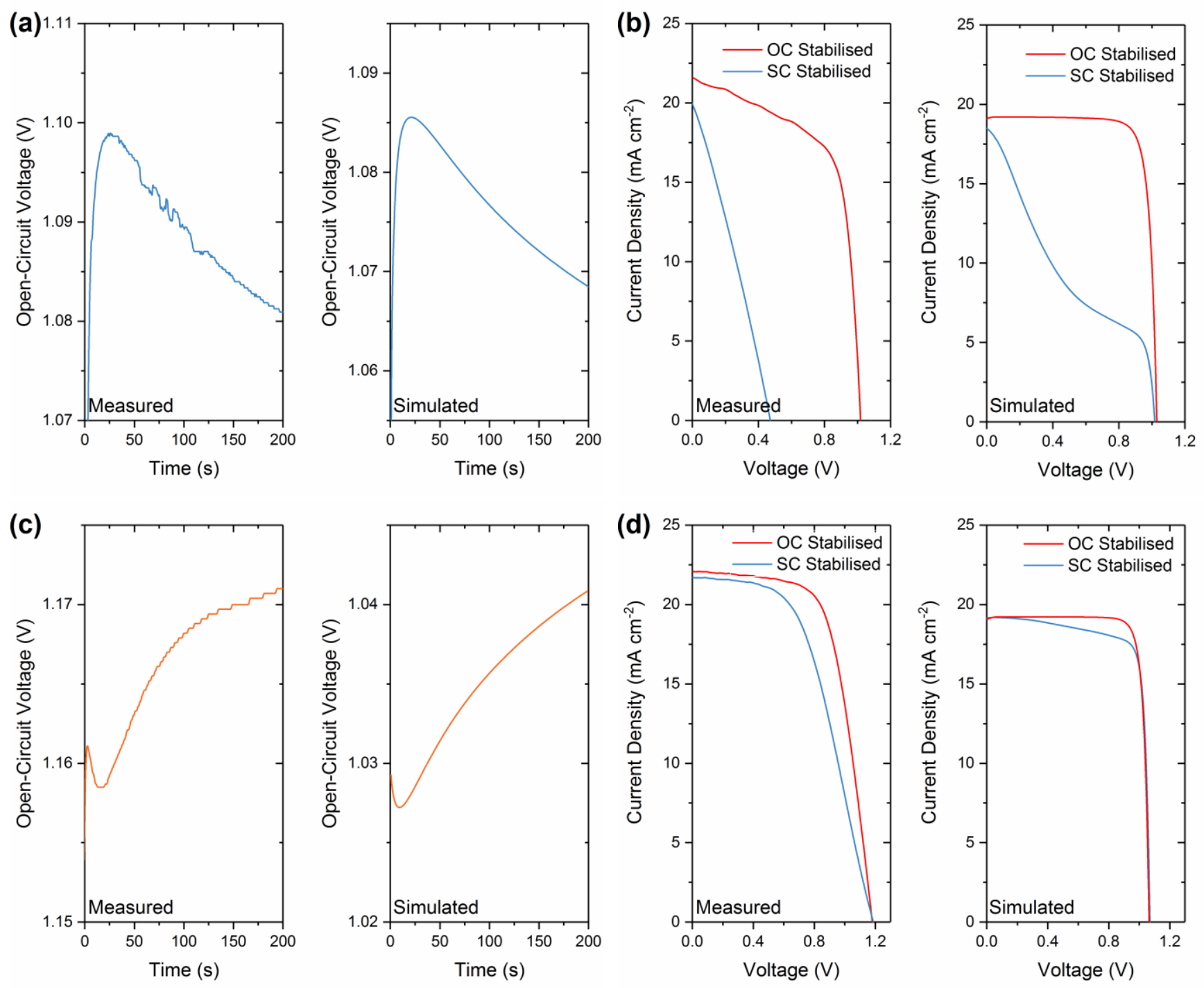

Figure 3: Experimental measurements and simulation of the open-circuit voltage transient following abrupt illumination from dark conditions for a $\mathrm{MAPbI}_{3}$ PSC (a), and a FA/MA/Cs/Rb-based PSC (c). Correspondingly, the J-V hysteresis measured after pre-biasing under illumination at open-circuit (OC) and short-circuit (SC) conditions (b and d) and sweeping the voltage at 10,000 mV/s. 

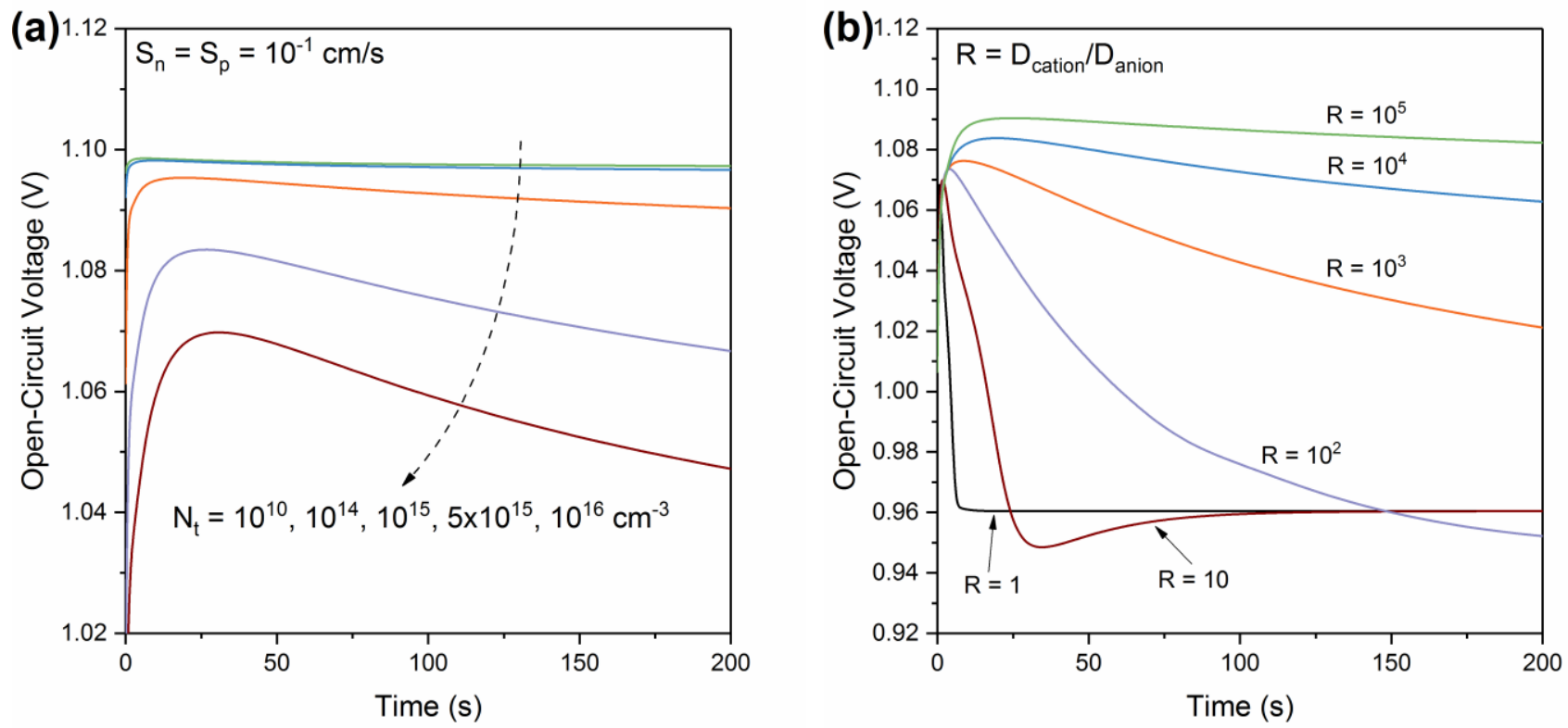

Figure 4: The influence of bulk trap defect concentration on the transient voltage response, in this example the replication of type A OCVT. With increasing bulk recombination (a) the voltage response begins to take on the characteristic non-monotonic rise and fall. This could be consistent with a recent study that observed the appearance of type A-like OCVTs in aged PSCs ${ }^{17}$. The ratio of ion diffusivity, $D_{\text {cation }} / D_{\text {anion }}$, for the cation and anion population is also highly influential on the OCVT (b).

\section{The interaction between net ion concentration and SRH recombination}

We have shown that mobile anions and cations in a transient drift-diffusion model can replicate two unusual OCVTs in PSCs, in addition to J-V hysteresis. In this section, we present a more detailed explanation of the interaction between ion migration, charge carrier distribution and SRH recombination that gives rise to time-varying open-circuit voltage in PSCs. This requires a closer look at the relative sensitivity of SRH, Auger and radiative recombination mechanisms to the electron-hole distribution.

We begin with a reminder that in this model ions influence electron and hole dynamics solely through their electric charge. Ions enter the drift-diffusion semiconductor model through the charge density equation (eq. 9 of supplementary information) in the same fashion as ionised dopant impurities. They do not function as trapping centres, and the concentration of recombination-active traps is therefore independent of the ion distribution. This is arguably the simplest possible implementation of ions in the 
semiconductor drift-diffusion model. Ions are therefore functionally equivalent to ionised dopant impurities, differentiated only by non-zero mobility. Consequently, the ions only influence the system where migration produces a net concentration of anions or cations, as is notably demonstrated by the formation of the ion double layer at the transport layer interfaces to screen the bulk electric field ${ }^{9,12}$.

The net ion distribution throughout the perovskite absorber begins to adjust following illumination, as the ion populations transition to a new steady-state. To illustrate this, it is useful to consider the two simulated distributions between which the ions migrate. Figure 5 plots the steady-state distribution of the cations and anions under open-circuit conditions in the dark and under illumination when both are mobile (as is the case in the simulations of this work), or when only the cation is mobile (as has often been considered). With only mobile cations, the accumulation and depletion at the respective interfaces is predominantly limited to the narrow Debye layer at the interface regions (Figure 5b and 5d). Illumination of the cell reduces the bulk electric field and accordingly reduces the steady-state accumulation. By comparison, when both ions are mobile, in the dark there is significant segregation of both ions towards opposite interfaces: cations toward the HTL, and anions to the ETL (Figure 5a and 5c). This is driven by the assumption that compensation of the electric field takes place entirely in the perovskite absorber as a result of the metal-semiconductor interfaces. Under illumination and a reduced bulk electric field, the ions accumulate predominantly in narrow layers at the interface, while approaching (although not quite reaching) equal concentrations through the bulk. 

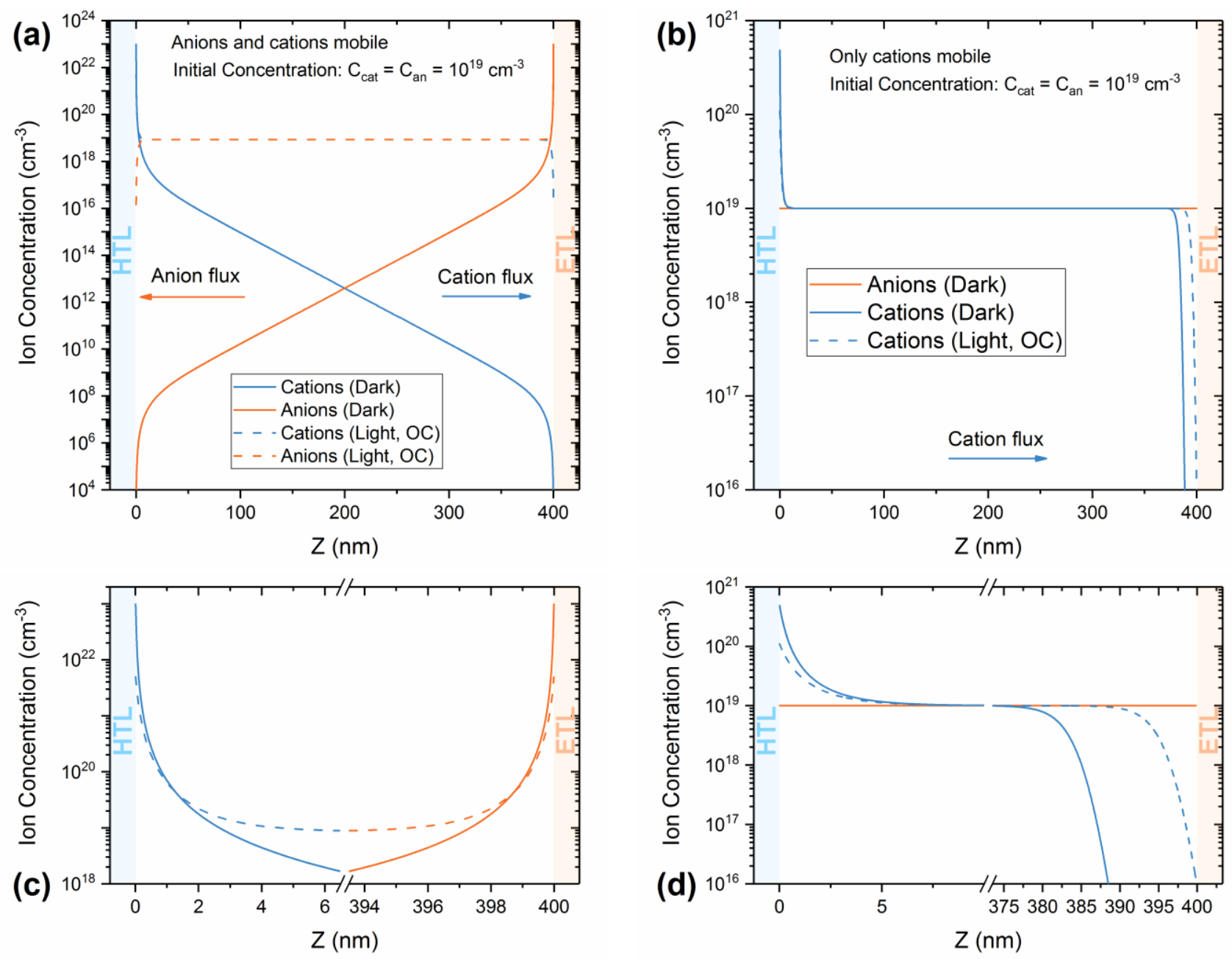

Figure 5: Steady-state distribution of anions and cations under the initial dark condition (solid lines) and final illuminated condition (dashed lines), showing the two distributions between which the ion population transitions after illumination. Shown are the distributions where both anions and cations $(\mathrm{a}, \mathrm{c})$ or only cations (b,d) are mobile. Figures (c) and (d) show magnified views of the near-interface regions at which the ions accumulate. The arrows indicate the direction of ion flux during the transition between the dark and light distributions (i.e. during the OCVT measurement). In (a) the concentration of anions and cations under light are roughly equal through the bulk. Note $\mathrm{x}$-axis break in (c) and (d).

As the net ion population evolves between these two distributions, the changing profile of the net ion concentration has a significant impact on the distribution of electrons and holes. This is shown in Figure 6, which plots the spatial distribution of net ionic charge, the electron-hole densities and the recombination rate at the peak of the type A OCVT. At this point, the cell has begun its relaxation from 
the dark ion conditions in Figure 5a. Illumination of the cell, held at open-circuit, reduces the magnitude of the bulk electric field, prompting migration of the cation and anion populations away from their accumulation at the HTL and ETL interfaces, respectively. The cations are approximately four orders-ofmagnitude more diffusive in this example, and the rapid cation migration to the ETL creates a cation surplus through most of the perovskite bulk. This attracts an equivalent concentration of electrons to maintain charge neutrality. The majority of the perovskite bulk is thus effectively n-type. The law of mass action dictates that the population of holes is suppressed, such that $n p=n_{i}^{2}\left(\exp \left(\Delta \eta / V_{t}\right)-1\right)$, where $\Delta \eta$ is the separation of the quasi-Fermi levels, and $V_{t}$ the thermal voltage. In addition, at the interfaces the band bending accumulates a large concentration of holes at the HTL, and electrons at the ETL. The balance of these forces with charge transport in the perovskite bulk results in the distribution of electrons and holes of Figure 6.

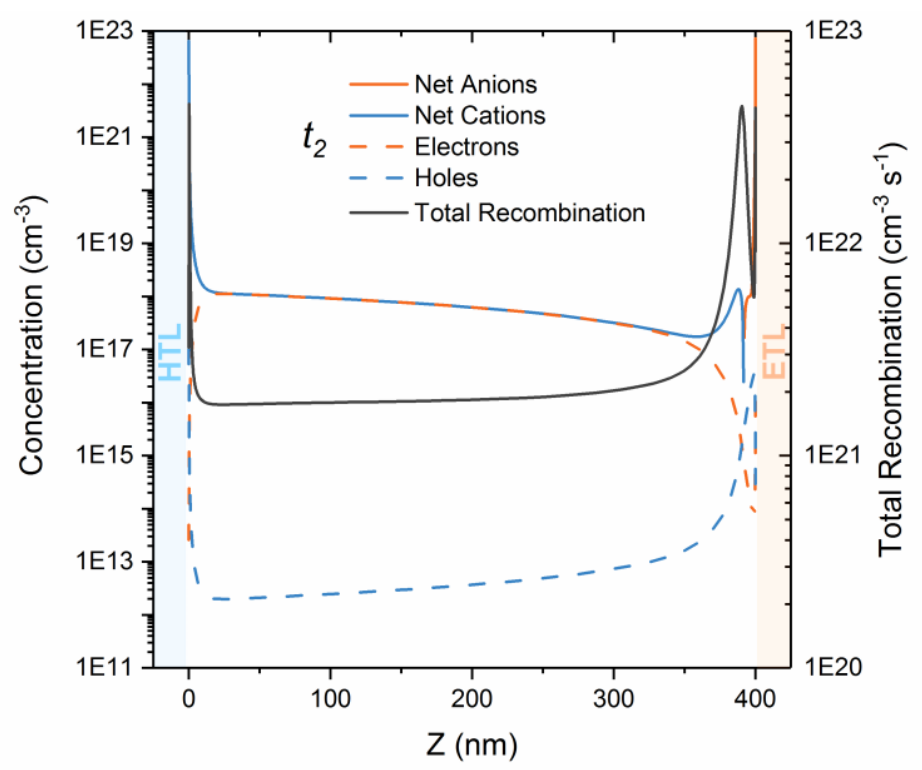

Figure 6: Spatial distribution of electrons and holes, net ions and the corresponding distribution of total recombination at time $t_{2}$ in the type ACVT (with reference to Figure 7a).

The distribution of recombination changes with the electron-hole population. Where the concentration of electrons and holes converge near the interfaces of the perovskite absorber, the rate of 
recombination increases, most notably at the near-surface region toward the ETL. In the bulk, where the electron-hole asymmetry is greatest, recombination is relatively suppressed. As the ion population continues to evolve to the new steady state, the net ion distribution changes, and so too does the distribution of electrons and holes and the distribution of recombination.

Trap-mediated (SRH) recombination is the primary influence on the distribution and magnitude of recombination. Relative to intrinsic recombination mechanisms, SRH recombination can be highly sensitive to the asymmetry of the electron-hole population. To illustrate this, in Figure 7 we compare key properties of the perovskite absorber during type A OCVT both with and without SRH recombination, while Figure 8 plots the corresponding evolution of the energy bands. We plot the spatial distribution of the net ion and electron-hole concentrations, recombination and quasi-Fermi levels at several points along the OCVT profile, along with the final distribution at the ion new steady-state (dashed lines, figure 5a).

The first observation is that the presence or absence of SRH recombination has only a small effect on ion migration. The net concentration of ions at each time point under both conditions is roughly equivalent. What is significantly affected, however, is the distribution of local recombination. From their mathematical forms (equations $1-3$ in supplementary information), we see that all recombination mechanisms are sensitive to the electron-hole $(n p)$ product. Indeed, radiative recombination is sensitive only to the $n p$ product. Auger and SRH recombination, by comparison, are sensitive also to the relative concentration of electrons and holes.

The simulation computes that the $n p$ product varies little across the width of the cell at all times in the voltage transient. Consequently, the rate of radiative recombination is also roughly spatially constant, and is insensitive to the redistribution of net ions. The picture looks different when we consider Auger or SRH recombination. These mechanisms are (functionally) distinct from radiative recombination in that they are sensitive to both the $n p$ product and the relative concentration of electrons and holes. Although 
the $n p$ product is roughly uniform, the relative electron-hole concentrations vary substantially. For example, with recombination-active traps distributed uniformly in the absorber, the majority of recombination occurs toward the ETL interface (Figure 7d), where the electron and hole populations converge (Figure 7c). Although Auger recombination is also sensitive to the ratio of the electron and hole concentration, the rate of recombination is too small to produce the observed effects at the carrier densities expected under normal operating conditions (as shown by the rapid stabilisation of the OCVT without SRH recombination in Figure 7f). This leaves SRH recombination as the dominant recombination process when non-monotonic voltage transients are recorded. It also describes the perovskite absorber as a system in which it is SRH recombination that reveals the movement of ions (evidence for which was provided by Calado et $\left.a l^{10}\right)$.

However, specifically, the SRH model is sensitive to the ratio of the fundamental lifetime-charge density product for electrons and holes (that is, $n \tau_{n} / p \tau_{p}$ ). In this analysis, we have assumed symmetric capture cross sections, and therefore symmetric fundamental lifetimes (i.e. $\tau_{n}=\tau_{p}$ ). A divergence in the fundamental lifetimes (and also, the trap energy level) affects the sensitivity of the SRH model to the relative charge carrier densities, which in turn can affect the characteristic of the simulated OCVT. Type A and B OCVTs can be replicated under different parameterisations of the trap population, but the possible range of implementations is beyond the scope of this work. The approach described above was taken to emphasize the potential influence of SRH recombination.

At a broader level, the interaction between ion migration and cell voltage described by Figure 7 can be thought of as the perovskite absorber maintaining balance between generation and recombination. Generation throughout the measurement is fixed, and at open-circuit, there is zero charge extraction. Therefore, total recombination must be constant and equal to total generation. We have shown that when the spatial net ion concentration changes with time during ion migration, non-radiative recombination modelled after SRH statistics can lead to a significant change in recombination rates. Consequently, the 
quasi-Fermi level separation must adjust (which in turn adjusts the $n p$ product and the voltage measured at the cell terminals) in order to maintain equilibrium between generation and recombination. We note that this explanation is consistent with conditions in which the OCVT is the result of dominant SRH recombination at the perovskite-transport layer interface(s). Then, surface traps, with recombination modelled after SRH statistics, are required to produce the observed effects due to an equivalent interplay of net ion concentration and SRH recombination at the surface.

The influence of the net ionic charge density on device voltage has a direct analogy in the impact of net doping (that is, $\left|\mathrm{N}_{\mathrm{A}}-\mathrm{N}_{\mathrm{D}}\right|$ ) on silicon semiconductor solar cells. It is well understood that changes in the relative surplus or deficit of one dopant impurity over another changes the relative concentration of electrons and holes and thus has significant impact on charge carrier lifetimes (see Macdonald et al ${ }^{36}$ ). The functional form of ions in this perovskite model is directly equivalent to ionised dopant impurities, and therefore the influence of the time-varying net ion distribution plays out in a similar fashion.

Finally, the type B OCVT has a characteristic form similar to the non-monotonic transition between fast and slow components of open-circuit voltage decay measurements measured by Pockett et $a l^{34}$. We do not consider voltage decay in this work, but it is a natural extension to expect that the relaxation of ions after the removal of illumination will have a similar effect on recombination during voltage decay. The similarity in the non-monotonic response observed in reference ${ }^{34}$ over a similar period of seconds may support this conclusion. 

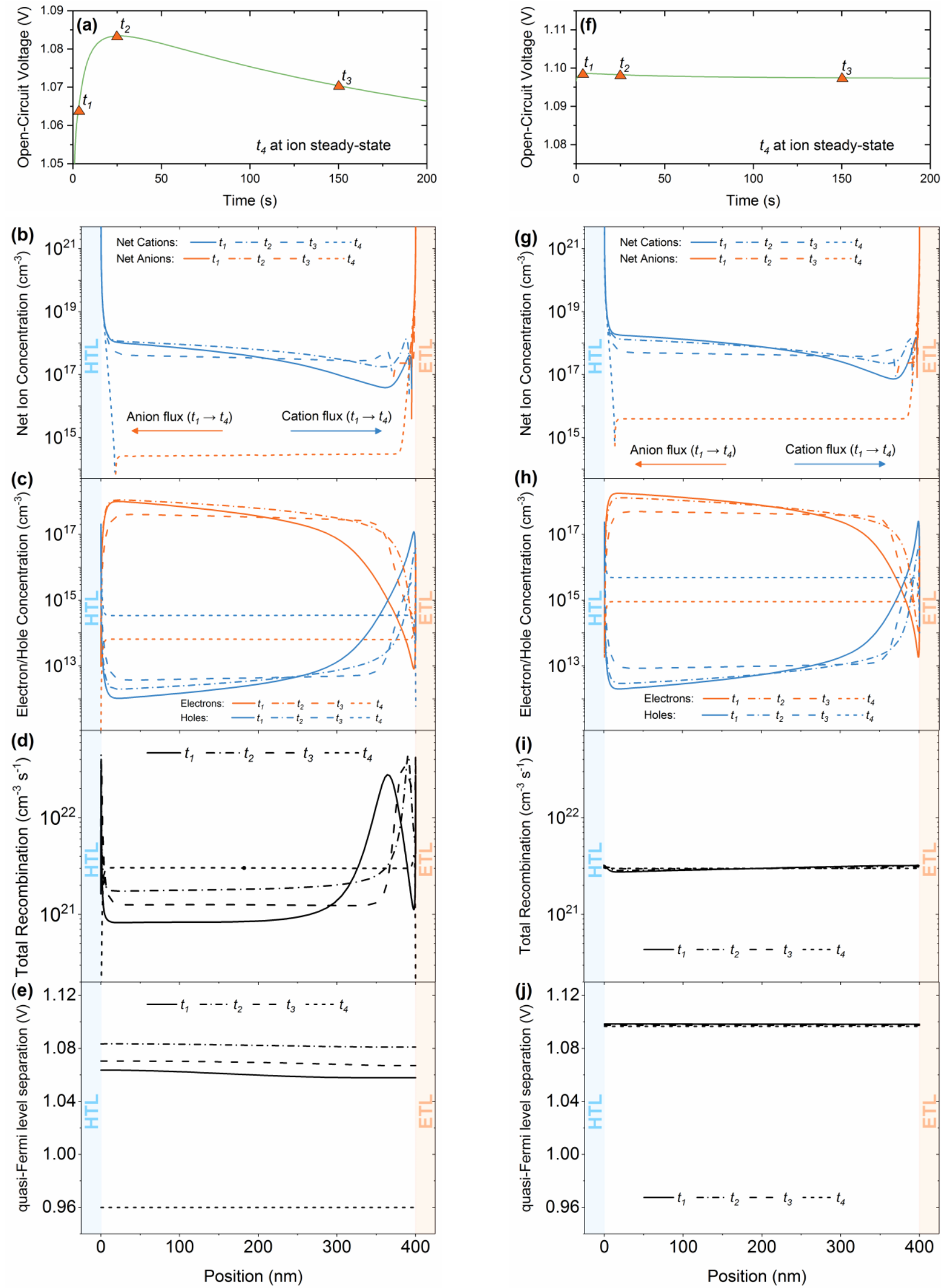
Figure 7: Spatial distribution of net ion concentration, electron-hole concentrations, total recombination rate, and quasi-Fermi level separation through the perovskite absorber for (right column) a device without SRH recombination (i.e. only intrinsic processes: radiative and Auger recombination), and (left column) with SRH recombination through a uniform distribution of trap defects. The time points, $t_{1} \rightarrow t_{4}$ are as marked on the uppermost OCVT plots. The final point, $\mathrm{t}_{4}$, is computed at the ion steady-state (not shown, see next section for discussion). The arrows in figures $b$ and $g$ indicate the direction of ion migration during the voltage transient. The simulated OCVT is shown for the device with (a) SRH recombination, and without (f) SRH recombination.
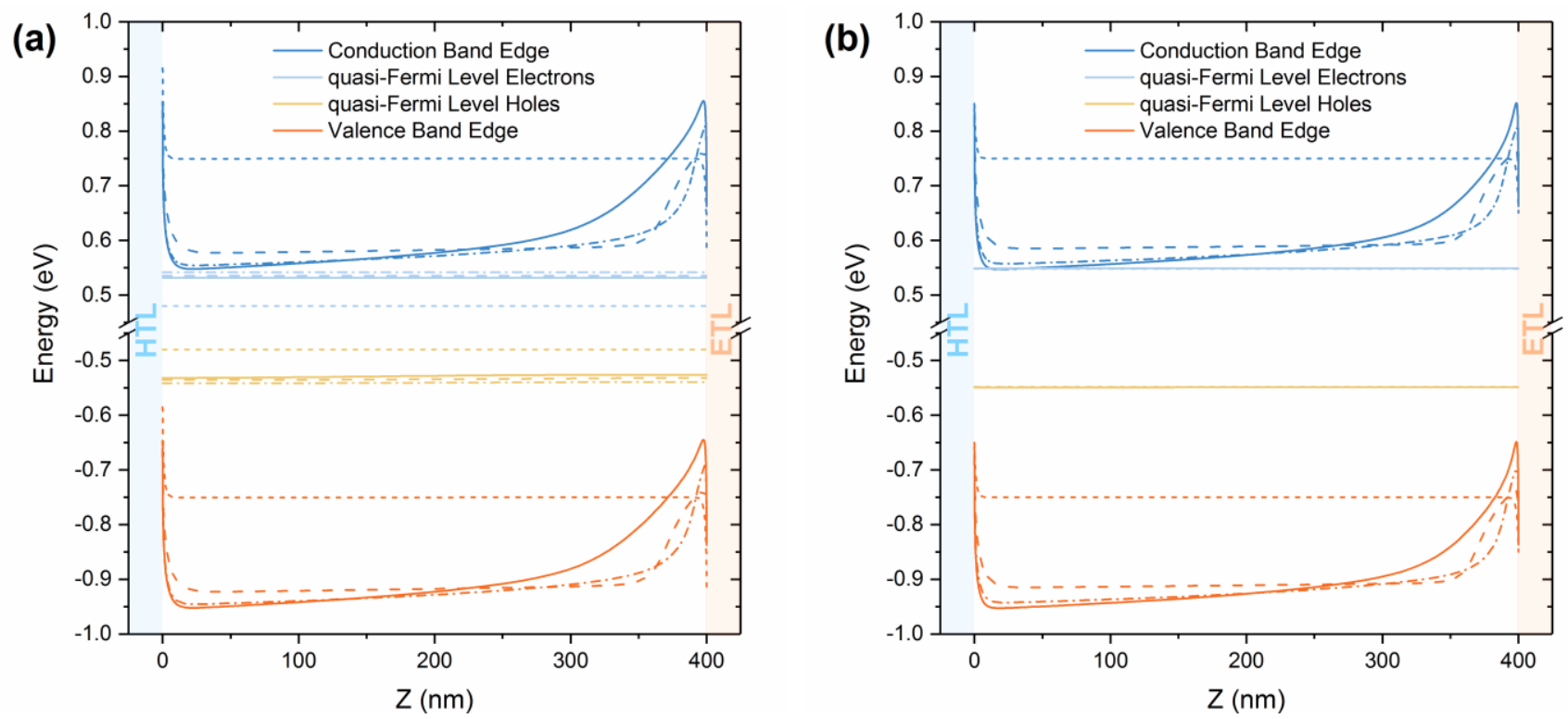

Figure 8: Spatial distribution of the energy levels in the perovskite absorber, with SRH (a) and without SRH (b) recombination. In (b), the quasi-Fermi levels for electrons and holes are unchanged throughout the simulation, and overlap in the figure. The line styles represent the time point of the simulation as per the definitions in Figure 7. Note y-axis break.

\section{Observations on prolonged OCVTs}

We conclude by exploring when the steady-state of the ion population is truly achieved where the 'slow' ion has a diffusivity up to four orders of magnitude slower than the 'fast' ion. It is unlikely that the slow, second ion reaches steady-state within the period presented in the analysis of Figure 3 . Thus, in Figure 9, we plot an extended simulation of type A and B OCVT. The simulation predicts that, for these 
ion diffusivities, full steady-state for both anions and cations is not achieved until roughly $2 \times 10^{5} \mathrm{~s}(2.5$ days) for both OCVTs. In both cases, the simulation predicts additional maxima and/or minima in the voltage transient. In type A, the relaxation to steady state appears predominantly as a gradual fall in cell voltage, with a local minimum before steady-state. However, for type B, we predict an additional maximum and minimum in voltage before steady-state. We should be cautious in interpreting these results, as the time constants of the response and the point of eventual steady-state in both time and voltage vary with many material and device parameters about which we can only make approximations, including the relative permittivity, ion concentration and diffusion coefficients, the SRH lifetime and even the absorber thickness. However, the simulations indicate that even in the absence of permanent degradation, slowmoving ions may influence the measured voltage over periods of hours to days. This response may easily be misinterpreted as degradation, when instead it could be evidence of reversible ion migration. This is in agreement with recent results that indicated that slow, reversible degradation may be the migration of a slow-moving cation over a period of hours ${ }^{37}$.

Experimental verification of these predictions is challenging. The model suggests that the OCVTs depend on a substantial amount of SRH recombination, which may be correlated with already degraded or otherwise unstable devices. In addition, a prolonged measurement is likely to see the superposition of permanent degradation or modification of the perovskite and transport layers (e.g. $\mathrm{Li}^{+}$ingress from SpiroOMeTAD $^{38}$, or transport layer delamination ${ }^{39}$ ). It is also possible that influential properties, such as the trap density, will be time-variant as the cell ages. Low hysteresis, high stability cells would not be expected to produce these transients due to reduced recombination. Nonetheless, in Figures 9c and 9d we plot experimental data of prolonged measurements of type A and B OCVTs. In this case, the type B OCVT was measured on a quadruple-cation (including $\mathrm{Rb}$ ) cell with an mp- $\mathrm{TiO}_{2} \mathrm{ETL}$ and a copper thiocyanate HTL. The type A response is the extended measurement of figure 3a. For the type A OCVT of the $\mathrm{MAPbI}_{3}$ cell, we find that the voltage transient closely approximates the residual decay out to 2,000s. Although it 
is possible to replicate the general form of type A transience with only one mobile ion ${ }^{10,33}$, we found that we could not replicate agreement with the time constants of the decay over this prolonged period without the additional, slower anion.

For the quadruple-cation cell, we observe that the experimental OCVT shows the second, maximum turning point predicted by the model (Figure 8d). Beyond this point the cell voltage continued to fall for several hours, a notable digression from the model. However, at the end of the measurement we verified the cell performance was permanently degraded. This suggests that even the unusual predictions of Figure $8 \mathrm{~b}$ may capture relevant processes occurring in the cell, until the point of degradation.
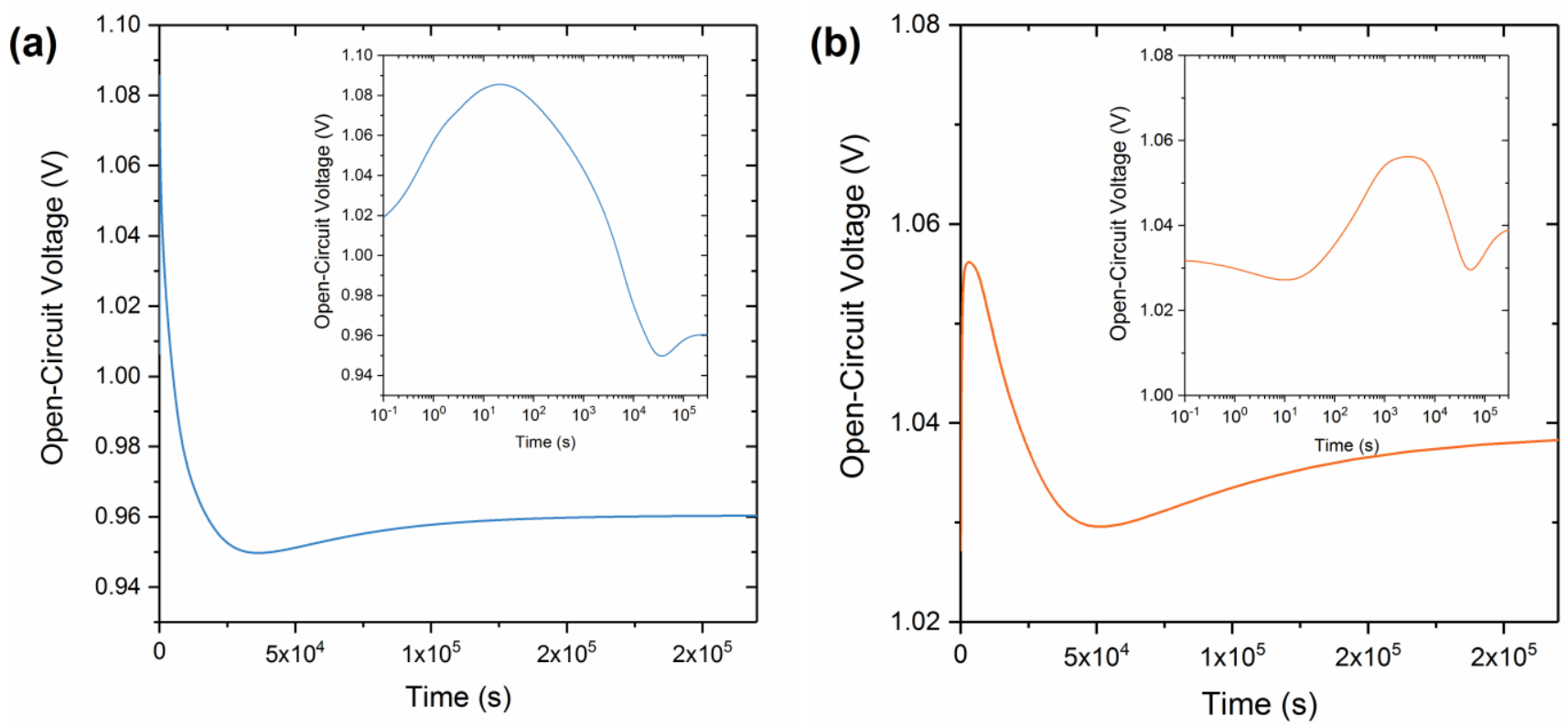

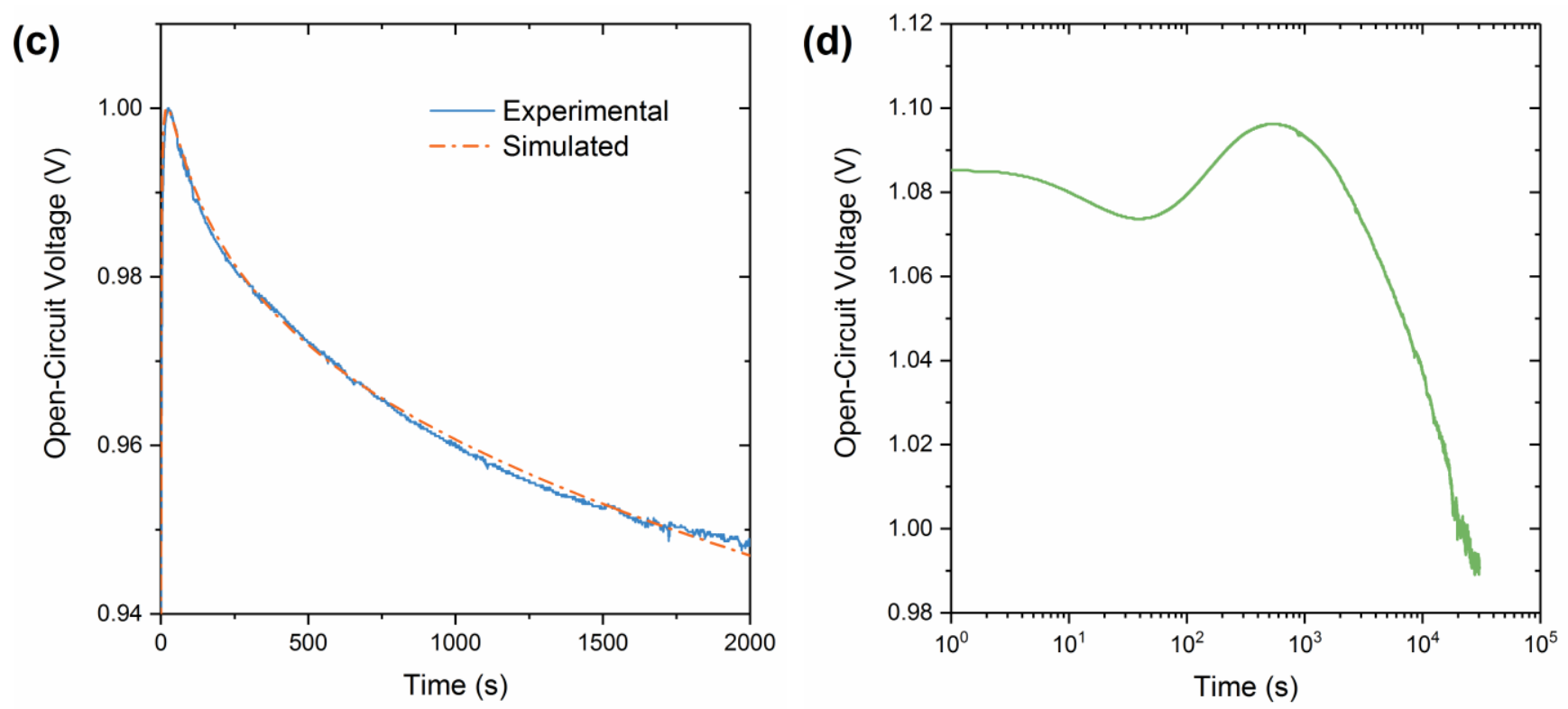

Figure 9: Simulated long-term voltage response of Type A OCVT (a), and Type B OCVT (b). Inset figures plot the transient response on a $\log (t)$ scale. The Type A transient is replicated closely by the simulation out to 2,000s (c). For the type B OCVT, a prolonged measurement of an Rb-containing quadruple-cation cell (d), replicates the second maximum turning point predicted by the simulation (b), but disagrees with the final, minimum turning point, possibly a result of cell degradation.

\section{CONCLUSION}

This work has employed a thorough transient numerical model of perovskite semiconductors to describe the interaction of a model of ion migration and non-radiative recombination in a perovskite absorber. We have provided a mechanistic explanation of the close interaction between ion migration and electron-hole recombination and therefore illustrated how the electric charge of mobile ions can have nonintuitive, non-monotonic effects on the transient voltage response. Our model predicts that the migration of ions can create net concentration densities at not only the interface of the perovskite layer, but also throughout the bulk. This in turn redistributes the electron-hole population asymmetrically, which significantly affects local recombination rates through trap-mediated $(\mathrm{SRH})$ recombination. Through this interaction, the open-circuit voltage can fluctuate non-monotonically to maintain balance between 
generation and recombination, when a change in excitation prompts the migration of ions to a new steadystate distribution.

It is notable that our implementation of relatively simple physical models of charge transport can replicate non-monotonic voltage transients as well as hysteretic $\mathrm{J}-\mathrm{V}$ curves. The multiple timescales of the voltage transients may have pointed towards multiple physical processes. Instead, we have shown that these responses can be replicated through the subtle interaction of conventional models of semiconductor charge transport and the migration of ionic charge. Moreover, we found that two mobile ions (an anion and a cation population) may be necessary to replicate both the characteristic and time scales of the voltage transients. The characteristics of the response are predicted to be highly sensitive to the relative diffusion coefficients of the ion populations. This may, in future, provide some diagnostic utility to this relatively simple measurement protocol, although for the time being we caution that there is still uncertainty about the value of many influential parameters.

We conclude by echoing a recent call ${ }^{11}$ to carefully consider the impact of mobile ionic charge before proposing additional hypotheses for hysteretic behaviour in perovskite solar cells. In addition, our results depend on the unique dependence of SRH recombination on the relative charge carrier density, a relationship that is not represented in injection-independent rate constants often used to approximate recombination kinetics in perovskite cells. This motivates the continued use of numerical device models for PSCs, which can capture the injection-dependence of recombination mechanisms and therefore the nuanced interaction with ion migration. 


\section{Acknowledgements}

This project received funding from the Australian Renewable Energy Agency ARENA and the Australian Centre for Advanced Photovoltaics (ACAP). The views expressed are not necessarily the views of the Australian Government, which does not accept responsibility for any of the information or advice contained herein. The cooperation of Fraunhofer ISE and ANU was supported by the German Federal Ministry of Education and Research $(\mathrm{BMBF})$ within the German-Australian research cooperation cluster CCPV under contract number 01DR17019.

\section{Supporting Information}

Provides detail on the models used to compute the results presented in this work, and the device parameters. Discussion of simulation results under conditions of dominant interface recombination and mobile ions. 
(1) Snaith, H. J.; Abate, A.; Ball, J. M.; Eperon, G. E.; Leijtens, T.; Noel, N. K.; Stranks, S. D.; Wang, J.; Wojciechowski, K.; Zhang, W. Anomalous Hysteresis in Perovskite Solar Cells. J Phys Chem Lett 2014, 5, 1511-1515.

(2) Hoke, E. T.; Slotcavage, D. J.; Dohner, E. R.; Bowring, A. R.; Karunadasa, H. I.; McGehee, M. D. Reversible Photo-Induced Trap Formation in Mixed-Halide Hybrid Perovskites for Photovoltaics. Chem. Sci. 2014, 6, 613-617.

(3) Yuan, Y.; Chae, J.; Shao, Y.; Wang, Q.; Xiao, Z.; Centrone, A.; Huang, J. Photovoltaic Switching Mechanism in Lateral Structure Hybrid Perovskite Solar Cells. Adv. Energy Mater. 2015, 5, 1500615.

(4) Xiao, Z.; Yuan, Y.; Shao, Y.; Wang, Q.; Dong, Q.; Bi, C.; Sharma, P.; Gruverman, A.; Huang, J. Giant Switchable Photovoltaic Effect in Organometal Trihalide Perovskite Devices. Nat. Mater. 2015, 14, 193-198.

(5) Yang, T.-Y.; Gregori, G.; Pellet, N.; Grätzel, M.; Maier, J. The Significance of Ion Conduction in a Hybrid Organic-Inorganic Lead-Iodide-Based Perovskite Photosensitizer. Angew. Chem. 2015, 127, 8016-8021.

(6) Eames, C.; Frost, J. M.; Barnes, P. R.; C, O., Brian; Walsh, A.; Islam, S. M. Ionic Transport in Hybrid Lead Iodide Perovskite Solar Cells. Nat Commun 2015, 6, 7497.

(7) M. Azpiroz, J.; Mosconi, E.; Bisquert, J.; Angelis, F. D. Defect Migration in Methylammonium Lead Iodide and Its Role in Perovskite Solar Cell Operation. Energy Environ. Sci. 2015, 8, 21182127.

(8) Haruyama, J.; Sodeyama, K.; Han, L.; Tateyama, Y. First-Principles Study of Ion Diffusion in Perovskite Solar Cell Sensitizers. J. Am. Chem. Soc. 2015, 137, 10048-10051.

(9) van Reenen, S.; Kemerink, M.; Snaith, H. J. Modeling Anomalous Hysteresis in Perovskite Solar Cells. J. Phys. Chem. Lett. 2015, 6, 3808-3814.

(10) Calado, P.; Telford, A. M.; Bryant, D.; Li, X.; Nelson, J.; O’Regan, B. C.; Barnes, P. R. F. Evidence for Ion Migration in Hybrid Perovskite Solar Cells with Minimal Hysteresis. Nat. Commun. 2016, 7.

(11) Jacobs, D. A.; Wu, Y.; Shen, H.; Barugkin, C.; Beck, F. J.; White, T. P.; Weber, K.; Catchpole, K. R. Hysteresis Phenomena in Perovskite Solar Cells: The Many and Varied Effects of Ionic Accumulation. Phys. Chem. Chem. Phys. 2017.

(12) Richardson, G.; O’Kane, S. E. J.; Niemann, R. G.; Peltola, T. A.; Foster, J. M.; Cameron, P. J.; Walker, A. B. Can Slow-Moving Ions Explain Hysteresis in the Current-voltage Curves of Perovskite Solar Cells? Energy Environ. Sci. 2016, 9, 1476-1485.

(13) Neukom, M. T.; Züfle, S.; Knapp, E.; Makha, M.; Hany, R.; Ruhstaller, B. Why Perovskite Solar Cells with High Efficiency Show Small IV-Curve Hysteresis. Sol. Energy Mater. Sol. Cells 2017, 169, 159-166.

(14) Sherkar, T. S.; Momblona, C.; Gil-Escrig, L.; Bolink, H. J.; Koster, L. J. A. Improving Perovskite Solar Cells: Insights From a Validated Device Model. Adv. Energy Mater. 2017, 7, n/a-n/a.

(15) Stranks, S. D.; Burlakov, V. M.; Leijtens, T.; Ball, J. M.; Goriely, A.; Snaith, H. J. Recombination Kinetics in Organic-Inorganic Perovskites: Excitons, Free Charge, and Subgap States. Phys. Rev. Appl. 2014, 2, 034007.

(16) O’Regan, B. C.; Barnes, P. R. F.; Li, X.; Law, C.; Palomares, E.; Marin-Beloqui, J. M. Optoelectronic Studies of Methylammonium Lead Iodide Perovskite Solar Cells with Mesoporous $\mathrm{TiO}_{2}$ : Separation of Electronic and Chemical Charge Storage, Understanding Two Recombination Lifetimes, and the Evolution of Band Offsets during $J-V$ Hysteresis. $J$. Am. Chem. Soc. 2015, 137, 5087-5099. 
(17) Tress, W.; Yavari, M.; Domanski, K.; Yadav, P.; Niesen, B.; Baena, J. P. C.; Hagfeldt, A.; Graetzel, M. Interpretation and Evolution of Open-Circuit Voltage, Recombination, Ideality Factor and Subgap Defect States during Reversible Light-Soaking and Irreversible Degradation of Perovskite Solar Cells. Energy Environ. Sci. 2017.

(18) Gottesman, R.; Lopez-Varo, P.; Gouda, L.; Jimenez-Tejada, J. A.; Hu, J.; Tirosh, S.; Zaban, A.; Bisquert, J. Dynamic Phenomena at Perovskite/Electron-Selective Contact Interface as Interpreted from Photovoltage Decays. Chem 2016, 1, 776-789.

(19) Hu, J.; Gottesman, R.; Gouda, L.; Kama, A.; Priel, M.; Tirosh, S.; Bisquert, J.; Zaban, A. Photovoltage Behavior in Perovskite Solar Cells under Light-Soaking Showing Photoinduced Interfacial Changes. ACS Energy Lett. 2017, 2, 950-956.

(20) Duong, T.; Wu, Y.; Shen, H.; Peng, J.; Fu, X.; Jacobs, D.; Wang, E.-C.; Kho, T. C.; Fong, K. C.; Stocks, M.; et al. Rubidium Multication Perovskite with Optimized Bandgap for PerovskiteSilicon Tandem with over 26\% Efficiency. Adv. Energy Mater. 2017, 1700228.

(21) Saliba, M.; Matsui, T.; Domanski, K.; Seo, J.-Y.; Ummadisingu, A.; Zakeeruddin, S. M.; CorreaBaena, J.-P.; Tress, W. R.; Abate, A.; Hagfeldt, A.; et al. Incorporation of Rubidium Cations into Perovskite Solar Cells Improves Photovoltaic Performance. Science 2016, 354, 206-209.

(22) Bryant, D.; Wheeler, S.; O’Regan, B. C.; Watson, T.; Barnes, P. R.; Worsley, D.; Durrant, J. Observable Hysteresis at Low Temperature in "Hysteresis Free" Organic-inorganic Lead Halide Perovskite Solar Cells. J. Phys. Chem. Lett. 2015, 6, 3190-3194.

(23) deQuilettes, D. W.; Zhang, W.; Burlakov, V. M.; Graham, D. J.; Leijtens, T.; Osherov, A.; Bulović, V.; Snaith, H. J.; Ginger, D. S.; Stranks, S. D. Photo-Induced Halide Redistribution in Organic-inorganic Perovskite Films. Nat. Commun. 2016, 7, ncomms11683.

(24) Tress, W.; Marinova, N.; Moehl, T.; Zakeeruddin, S. M.; Nazeeruddin, M. K.; Grätzel, M. Understanding the Rate-Dependent J-V Hysteresis, Slow Time Component, and Aging in $\mathrm{CH}_{3}$ $\mathrm{NH}_{3} \mathrm{PbI}_{3}$ Perovskite Solar Cells: The Role of a Compensated Electric Field. Energy Env. Sci 2015, 8, 995-1004.

(25) Fell, A.; Walter, D.; Glunz, S. A Fast and Easy Perovskite Solar Cell Simulation Tool Featuring Ion Migration. In 33rd European Photovoltaic Solar Energy Conference and Exhibition; Amsterdam, Netherlands, 2017.

(26) J. O’Kane, S. E.; Richardson, G.; Pockett, A.; G. Niemann, R.; M. Cave, J.; Sakai, N.; E. Eperon, G.; J. Snaith, H.; M. Foster, J.; J. Cameron, P.; et al. Measurement and Modelling of Dark Current Decay Transients in Perovskite Solar Cells. J. Mater. Chem. C 2017, 5, 452-462.

(27) Shockley, W.; Read, W. T. Statistics of the Recombinations of Holes and Electrons. Phys. Rev. 1952, 87, 835-842.

(28) Macdonald, D.; Cuevas, A. Validity of Simplified Shockley-Read-Hall Statistics for Modeling Carrier Lifetimes in Crystalline Silicon. Phys. Rev. B 2003, 67, 075203.

(29) Fell, A.; Schön, J.; Schubert, M. C.; Glunz, S. W. The Concept of Skins for Silicon Solar Cell Modeling. Sol. Energy Mater. Sol. Cells 2017, 173, 128-133.

(30) Courtier, N. E.; Richardson, G.; Foster, J. M. A Fast and Robust Numerical Scheme for Solving Models of Charge Carrier Transport and Ion Vacancy Motion in Perovskite Solar Cells. ArXiv180105737 Phys. 2018.

(31) Courtier, N. E.; Foster, J. M.; O’Kane, S. E. J.; Walker, A. B.; Richardson, G. Systematic Derivation of a Surface Polarization Model for Planar Perovskite Solar Cells. ArXiv170809210 Phys. 2017.

(32) Brivio, F.; Butler, K. T.; Walsh, A.; van Schilfgaarde, M. Relativistic Quasiparticle SelfConsistent Electronic Structure of Hybrid Halide Perovskite Photovoltaic Absorbers. Phys. Rev. B 2014, 89 . 
(33) Wu, Y.; Shen, H.; Walter, D.; Jacobs, D.; Duong, T.; Peng, J.; Jiang, L.; Cheng, Y.-B.; Weber, K. On the Origin of Hysteresis in Perovskite Solar Cells. Adv. Funct. Mater. 2016, 26, 6807-6813.

(34) Pockett, A.; E. Eperon, G.; Sakai, N.; J. Snaith, H.; M. Peter, L.; J. Cameron, P. Microseconds, Milliseconds and Seconds: Deconvoluting the Dynamic Behaviour of Planar Perovskite Solar Cells. Phys. Chem. Chem. Phys. 2017, 19, 5959-5970.

(35) Xing, G.; Mathews, N.; Lim, S. S.; Yantara, N.; Liu, X.; Sabba, D.; Grätzel, M.; Mhaisalkar, S.; Sum, T. C. Low-Temperature Solution-Processed Wavelength-Tunable Perovskites for Lasing. Nat. Mater. 2014, 13, 476-480.

(36) Macdonald, D.; Cuevas, A. Recombination in Compensated Crystalline Silicon for Solar Cells. J. Appl. Phys. 2011, 109, 043704-043704-043708.

(37) Domanski, K.; Roose, B.; Matsui, T.; Saliba, M.; Turren-Cruz, S.-H.; Correa-Baena, J.-P.; Carmona, C. R.; Richardson, G.; Foster, J. M.; Angelis, F. D.; et al. Migration of Cations Induces Reversible Performance Losses over Day/Night Cycling in Perovskite Solar Cells. Energy Environ. Sci. 2017, 10, 604-613.

(38) Li, Z.; Xiao, C.; Yang, Y.; P. Harvey, S.; Hoe Kim, D.; A. Christians, J.; Yang, M.; Schulz, P.; U. Nanayakkara, S.; Jiang, C.-S.; et al. Extrinsic Ion Migration in Perovskite Solar Cells. Energy Environ. Sci. 2017, 10, 1234-1242.

(39) Soufiani, A. M.; Hameiri, Z.; Meyer, S.; Lim, S.; Tayebjee, M. J. Y.; Yun, J. S.; Ho-Baillie, A.; Conibeer, G. J.; Spiccia, L.; Green, M. A. Lessons Learnt from Spatially Resolved Electro- and Photoluminescence Imaging: Interfacial Delamination in $\mathrm{CH} 3 \mathrm{NH} 3 \mathrm{PbI} 3$ Planar Perovskite Solar Cells upon Illumination. Adv. Energy Mater. 2017, 7, 1602111. 


\section{TOC Graphic}

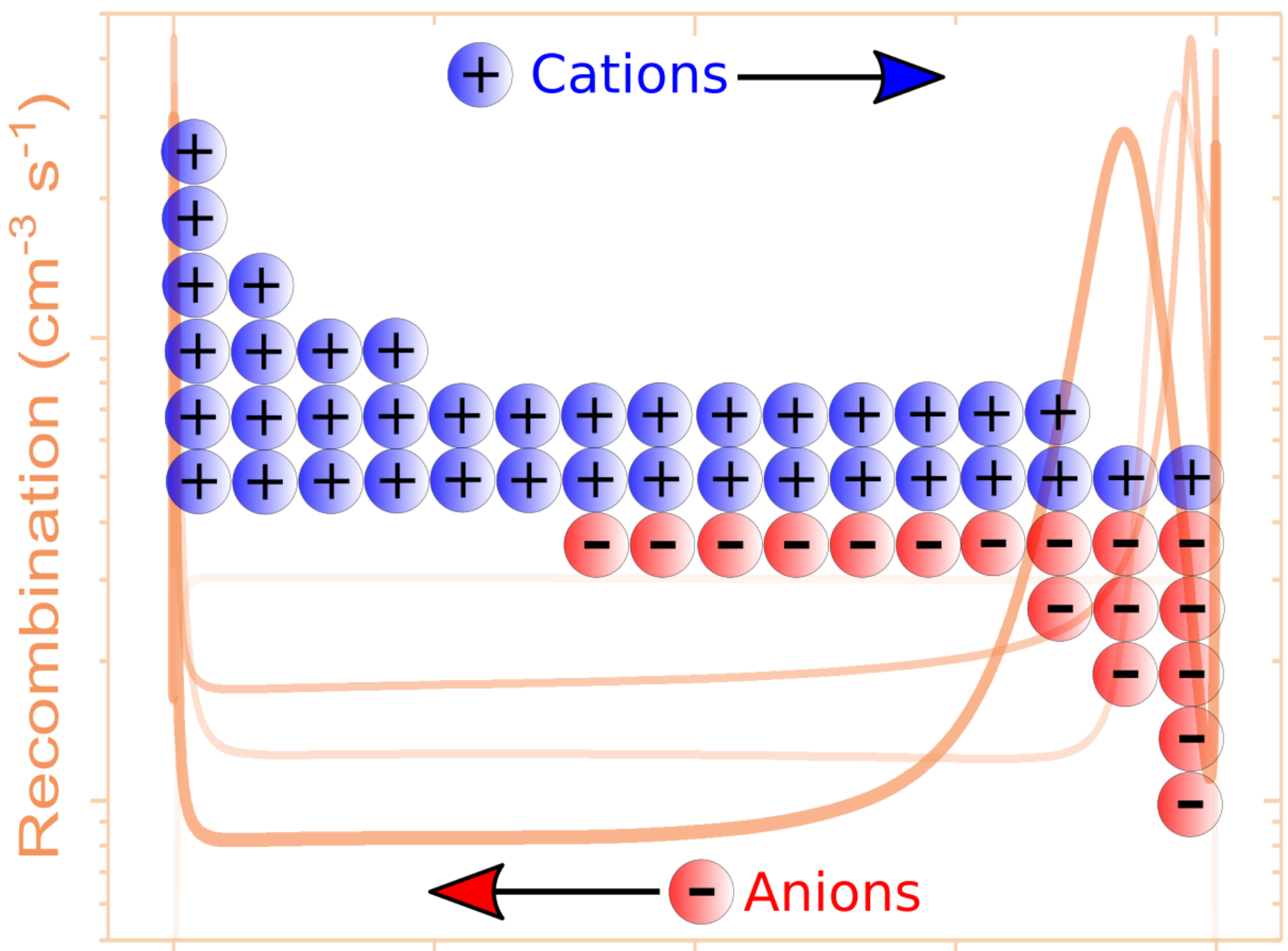

